\title{
INTERVENCIÓN COGNITIVO-CONDUCTUAL GRUPAL PARA PÉRDIDA DE PESO Y CALIDAD DE VIDA EN PACIENTES CANDIDATOS A CIRUGÍA BARLÁTRICA
}

\author{
MARIANA SIERRA MURGUÍA* \\ HOSPITAL GENERAL DE MÉXICO - DR. MANUEL GEA GONZÁLEZ \\ ARIEL VITE SIERRA \\ FACULTAD DE PSICOLOGÍA \\ UNIVERSIDAD NACIONAL AUTÓNOMA DE MÉXICO \\ MARGARITA TORRES TAMAYO \\ HOSPITAL GENERAL DE MÉXICO - DR. MANUEL GEA GONZÁLEZ
}

Recibido, noviembre 5/2012

Concepto evaluación, agosto 1/2013

Aceptado, noviembre 6/2013
Referencia: Sierra- Murguía, M., Vite- Sierra, A. \& Torres- Tamayo, M. (2014). Intervención cognitivoconductual grupal para pérdida de peso y calidad de vida en pacientes candidatos a cirugía bariátrica. Acta Colombiana de Psicología, 17(1), 25-34. doi: 10.14718/ ACP.2014.17.1.3

Resumen

\begin{abstract}
La obesidad es un problema de salud mundial asociado con el desarrollo de enfermedades crónico degenerativas. La cirugía bariátrica es el tratamiento que ha mostrado mayor efectividad para la obesidad severa; el paciente debe modificar su estilo de vida para mantener la pérdida de peso a largo plazo. Se ha visto que las intervenciones cognitivo conductuales han sido efectivas para lograr este cambio en el paciente. El presente estudio busca evaluar la efectividad de una intervención cognitivo conductual grupal en la pérdida de peso y calidad de vida antes de la cirugía. Se incluyeron nueve pacientes con obesidad mórbida candidatos a cirugía bariátrica sin comorbilidades psiquiátricas. Se realizó una evaluación inicial de calidad de vida que medía peso e IMC [Índice de Masa Corporal, por sus siglas en español]. Los pacientes fueron sometidos a una intervención grupal con el objetivo de promover la pérdida de peso mediante técnicas cognitivo conductuales. El promedio de pérdida de peso fue de $5.7 \mathrm{~kg}$ en las cinco semanas que duró el tratamiento, mostrando significancia estadística de .001; a su vez, con la pérdida de peso disminuyó el IMC con una significancia estadística de .002 . En cuanto a calidad de vida, hubo mejoría significativa en la percepción corporal y en la actitud al tratamiento.

Palabras clave: psicología bariátrica, cirugía bariátrica, obesidad, calidad de vida, cognitivo- conductual.
\end{abstract}

\section{COGNITIVE BEHAVIORAL GROUP INTERVENTION FOR WEIGHT LOSS AND QUALITY OF LIFE IN PATIENTS CANDIDATES FOR BARIATRIC SURGERY}

\begin{abstract}
Obesity is a global health problem associated with the development of chronic degenerative diseases. Bariatric surgery has proven to be the most effective treatment for severe obesity. Despite surgery, patients must change their lifestyle in order to maintain weight loss in the long term. It has been reported that cognitive behavioral interventions have been effective to promote a lifestyle change and weight loss in obese patients. The aim of this study was to assess the effectiveness of a cognitive behavioral group intervention on weight loss and quality of life before surgery. Participants were nine morbidly obese patients, without psychiatric comorbidities, candidates for bariatric surgery. An initial assessment of quality of life that measured weight and BMI [Body Mass Index, for its English acronym] was performed. Patients underwent a group intervention in order to promote weight loss through cognitive behavioral techniques. The average weight loss was $5.7 \mathrm{~kg}$ during the five weeks of treatment, showing statistical significance of .001. In turn, along with weight loss, there was a decrease in BMI, with a statistical significance of .002. Regarding quality of life, there was significant improvement in body perception and attitude toward treatment.

Key words: bariatric psychology, bariatric surgery, obesity, quality of life, cognitive-behavioral.
\end{abstract}

* Clínica de Obesidad, Hospital General “Dr. Manuel Gea González”, Calzada de Tlalpan 4800, sección XVI, CP 14080, México D.F. Tel. 40003000 Ext. 3372. mariana.sierra.m@gmail.com 


\title{
INTERVENÇÃO COGNITIVO-CONDUTUAL GRUPAL PARA PERDA DE PESO E QUALIDADE DE VIDA EM PACIENTES CANDIDATOS A CIRURGIA BARIÁTRICA
}

\author{
Resumo
}

\begin{abstract}
A obesidade é um problema de saúde mundial associado com o desenvolvimento de enfermidades crônico degenerativas. A cirurgia bariátrica é o tratamento que mostrou maior efetividade para a obesidade severa; o paciente deve modificar seu estilo de vida para manter a perda de peso a longo prazo. Observa-se que intervenções cognitivo conductuais têm sido efetivas para conseguir esta mudança no paciente. O presente estudo busca avaliar a efetividade de uma intervenção cognitivo conductual grupal na perda de peso e qualidade de vida antes da cirurgia. Incluíram-se nove pacientes com obesidade mórbida candidatos a cirurgia bariátrica sem co-morbilidades psiquiátricas. Realizou-se uma avaliação inicial de qualidade de vida que media peso e IMC [Índice de Massa Corporal]. Os pacientes foram submetidos a uma intervenção grupal com o objetivo de promover a perda de peso mediante técnicas cognitivo conductuais. A média de peso perdido foi de $5.7 \mathrm{~kg}$ nas cinco semanas que o tratamento durou, mostrando significância estatística de .001; ao mesmo tempo a perda de peso fez com que o IMC diminuísse com uma significância estatística de .002 . Com respeito à qualidade de vida, houve melhoria significativa na percepção corporal e na atitude com respeito ao tratamento.

Palavras chave: psicologia, bariátrica, cirurgia bariátrica, obesidade, qualidade de vida, cognitivo-condutual
\end{abstract}

\section{INTRODUCCIÓN}

La obesidad, declarada por la Organización Mundial de la Salud (OMS) como una epidemia (OMS, 2000), se encuentra asociada con el desarrollo de enfermedades como diabetes, hipertensión, cardiopatías, cáncer, entre otras (OMS, 2006). La OMS (2006) define la obesidad y el sobrepeso como una acumulación anormal o excesiva de grasa que puede ser perjudicial para la salud. Es originada por diversas causas biológicas, ambientales y conductuales, y conlleva numerosas complicaciones. Se identifica con el Índice de Masa Corporal (IMC), definido como la relación entre el peso y la talla $(\mathrm{kg} / \mathrm{m} 2)$, en donde se considera sobrepeso un IMC igual o superior a 25 , y obesidad un IMC igual o superior a 30 .

En las últimas décadas se han diseñado tratamientos encaminados a la pérdida controlada de peso, que pueden ser utilizados también para el control a largo plazo del mismo y para mantenerlo previniendo su recuperación (NIH, 1999).

\section{Los tipos de tratamiento son los siguientes:}

\section{Farmacológico}

El único fármaco aprobado actualmente por la FDA (Food and Drug Administration por sus siglas en ingles) para la reducción de peso es el Orlistat, indicado para pacientes con IMC $\geq 30 \mathrm{o} \geq 27$ si hay diabetes, dislipidemia o hipertensión. El Orlistat es un inhibidor reversible de las lipasas gastrointestinales, por lo que el paciente no puede hidrolizar a los triglicéridos de la dieta en ácidos grasos libres y monoglicéridos absorbibles. Debido a que los triglicéridos no hidrolizados no son absorbidos, el déficit calórico resultante tiene un efecto favorable sobre el control de peso; sin embargo, esto produce efectos secundarios gastrointestinales como manchas de grasa, flatulencia y secreción, urgencia fecal, heces con grasa/aceite, evacuación aceitosa, incremento en la defecación e incontinencia fecal, sobre todo cuando el paciente continúa ingiriendo alimentos con grasa. Estos efectos secundarios se han asociado a baja adhesión terapéutica, dejando limitado el tratamiento farmacológico para la obesidad.

\section{Quirúrgico}

Este procedimiento está indicado para pacientes con un IMC $\geq 40$ o $\geq 35$ en caso de comorbilidades producidas por la obesidad (American Association of Clinical Endocrinologists/The American Society/American Society of Metabolic and Bariatric Surgery, AACE/ TOS/ASMBS, 2008)

Se divide en tres tipos:

Restrictivo: Reducción del tamaño de la cámara gástrica limitando el consumo de alimentos.

Malabsortivo: Se reduce la longitud del intestino delgado disminuyendo la superficie de absorción de los nutrientes.

Mixto: Combina las dos anteriores. La más común es la derivación gastroyeyunal en $\mathrm{Y}$ de Roux o bypass gástrico.

Encaminados a modificación de hábitos y estilos de vida:

Tratamiento nutricional: Este tipo de tratamiento tiene como objetivo propiciar un balance calórico negativo (un déficit de 500 a $1.000 \mathrm{kcal}$ ) logrando que el paciente pierda en promedio el $8 \%$ del peso inicial entre tres y doce meses. Por esto debe disminuir la ingesta calórica diaria, así como el consumo de grasa saturada.

Actividad fisica: Contribuye a lograr un balance calórico negativo y es útil para el mantenimiento del peso deseado; 
reduce el riesgo cardiovascular y de diabetes. El programa de ejercicio debe realizarse de manera gradual.

Terapia conductual: Estrategias basadas en principios del aprendizaje, encaminadas a promover y mantener nuevas conductas saludables, así como a la disminución/ eliminación de conductas indeseables. En el paciente con obesidad las conductas predominantes son sedentarismo, sobreingesta, malos horarios de alimentación, exceso en el consumo de carbohidratos y grasas, así como pensamientos negativos asociados a la dieta, a la actividad física y a la capacidad del mismo sujeto para realizar esos cambios (baja autoeficacia). Estas conductas son las que se deben modificar y reestructurar para promover un estilo de vida saludable, y con ello la pérdida de peso (Van Dorsten et. al., 2008). Algunas de las técnicas psicológicas más utilizadas en el paciente con obesidad son: automonitoreo, control de estímulos, terapia de solución de problemas, manejo de contingencias, reestructuración cognitiva y apoyo social (involucrar a las personas cercanas del paciente) y prevención de recaídas (Van Dorsten et. al., 2008).

Terapia Combinada: Existe evidencia que indica que la combinación de restricción calórica junto con actividad física y terapia conductual es la más eficaz en la reducción y control de peso (Werrij, Jansen, Mulkens, Elgersma, Ament \& Hospers, 2009; Shaw, O'Rourke, Del Mar \& Kenardy, 2005).

Actualmente, el tratamiento que mayor efectividad ha mostrado para la obesidad severa a largo plazo es la cirugía bariátrica (Buchwald, 2005, Sjostrom, 2004). Para asegurar el éxito a largo plazo de esta cirugía, los pacientes deberán realizar cambios en su estilo de vida para lograr la pérdida de peso y mantenerla previniendo la recuperación gradual del peso; una proporción significativa de los pacientes que se someten a esta cirugía presenta dificultades para el mantenimiento de la pérdida de peso (Benotti y Forse, 1995). Por lo mismo, la motivación y la acción que lleva a un estilo de vida saludable debe ser elevada antes y después de la cirugía (Wild, Herzog, Wesche, Niehoff, Müller y Hain, 2009). A pesar de que la cirugía ha mostrado ser efectiva para la pérdida de peso, no ha mostrado poder modificar las conductas que llevan al paciente a una pérdida y mantenimiento de peso (Colquitt, Picot, Loveman y Clegg, 2009).

Diversos estudios (Buddeberg-Fischer, Klaghofer, Sigrist et al, 2004; Nicolai, Ippoliti, Petrelli, 2002; Hildebrant, 1998; Orth, Madan, Taddeucci, et al, 2008) indican que intervenciones tales como grupos psicoeducativos o de apoyo pueden mejorar los resultados de la cirugía bariátrica mediante la modificación conductual y ayudar a identificar aquellos pacientes que se encuentran en riesgo de desarrollar comorbilidades psiquiátricas.

Otro problema reportado es que existen pacientes con obesidad severa incapaces de tomar la decisión de someterse a una cirugía bariátrica o a algún otro método para reducción de peso. Generalmente, la historia de peso de estos pacientes se caracteriza por pérdida y recuperación del mismo de una manera cíclica, lo cual afecta la autoeficacia, la confianza y la habilidad para tomar decisiones, sobre todo, las relacionadas con la salud (Wild, et al. 2009). En el mismo estudio se realizaron sesiones de apoyo grupal donde surgieron algunos temas que preocupaban a los pacientes de manera recurrente, como la falta de autocontrol, el estigma relacionado con el peso, la posibilidad de un cambio, el deseo de mayor información relacionada con la cirugía bariátrica, el autocuidado, la autopercepción, la fobia social y el aislamiento actual. La intervención grupal sirvió de ayuda para pasar a la siguiente etapa de toma de decisiones relacionada con la cirugía y/o el programa de pérdida de peso. Este estudio concluye que las sesiones de apoyo grupal tienen efectos positivos sobre niveles de depresión, calidad de vida, motivación al tratamiento e índice de masa corporal (IMC), a pesar de que las sesiones no iban encaminadas a un programa de reducción de peso. Los autores proponen la intervención grupal como un tratamiento que puede ayudar a los pacientes con obesidad a reducir sintomatología depresiva y a aumentar la motivación al tratamiento.

Los puntos primordiales que deben tener los grupos de apoyo para estos pacientes incluyen varios factores, como los siguientes: a) siempre debe reforzarse la buena adhesión al tratamiento, así como los avances que vaya presentando el paciente, por mínimos que éstos sean; b) se debe brindar información y educación al paciente sobre los cambios conductuales que tendrá que realizar antes y después de la cirugía; c) permiten la identificación de problemas potenciales que se le pueden presentar al paciente y el desarrollo de estrategias de afrontamiento y resolución de éstos; d) proporcionan un ambiente seguro para el paciente y una oportunidad de que asuma los retos que le presenta la cirugía, al estar en compañía de otras personas que viven experiencias similares; e) por último, el paciente que está considerando la cirugía debe sentirse aceptado e integrado en el grupo para que éste funcione como una oportunidad de aprender de las personas que ya han pasado por dicha experiencia (Algazi, 2000).

La participación en grupos psicoeducativos para pacientes bariátricos ayudará a que los cuidados post-quirúrgicos sean más fáciles, tanto para éstos, como para el equipo multidisciplinario que los trata. Además, les proporciona 
una percepción de pertenencia y de apoyo, factores que influyen de manera positiva en la adhesión terapéutica; de igual manera, un grupo propicia la expresión de emociones relevantes y disminuye el estrés; por lo tanto, tiene el potencial de mejorar la adaptación del paciente al nuevo estilo de vida requerido por la cirugía bariátrica (Marcus y Elkins, 2004). Se ha reportado una correlación positiva entre el número de sesiones grupales a las que se asiste con la pérdida de peso post quirúrgica (Kaiser, et al, 2011).

De acuerdo con lo anteriormente expuesto el objetivo de la investigación fue: evaluar la efectividad de una intervención cognitivo conductual grupal en pacientes candidatos a cirugía bariátrica sobre la pérdida de peso y calidad de vida antes de la cirugía.

\section{MÉTODO}

\section{Participantes}

Se invitó a participar a pacientes que ingresaron a la clínica de obesidad del Hospital General "Dr. Manuel Gea González", durante el período comprendido entre enero y marzo de 2012. Se escogieron aquellos pacientes que no tenían contraindicaciones para cirugía, es decir, se realizó un muestreo por conveniencia. Se incluyeron 15 pacientes, de los cuales el $66 \%(n=10)$ eran mujeres y el $33 \%(n=5)$ hombres. Los participantes no cumplían criterios de psicopatología ni contraindicaciones psicológicas para cirugía diferentes a la falta de conocimiento sobre el procedimiento o expectativas poco realistas sobre los resultados de la cirugía. Todos los pacientes se encontraban en tratamiento multidisciplinario que incluía especialistas en nutrición, endocrinología, cirugía y cardiología. De los 15 pacientes que participaron en el estudio, seis no lo completaron por lo que debieron ser excluidos del mismo. De los nueve

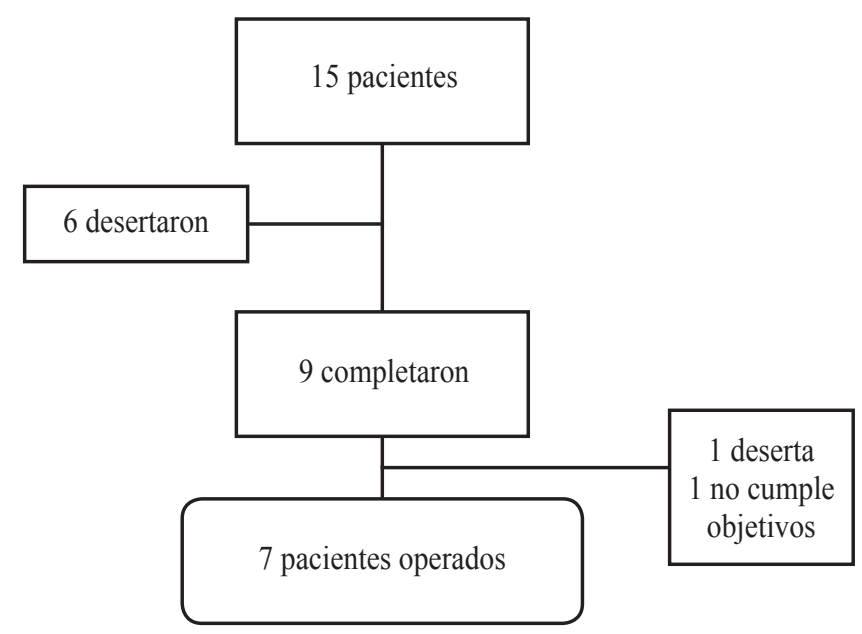

Figura 1. Diagrama de Flujo de los pacientes durante el estudio que lo completaron, dos no se operaron, una por falta de recursos económicos, y otro debido a que no cumplió los objetivos del tratamiento.

Se describen a continuación los datos sociodemográficos de los pacientes que completaron el estudio: $77.8 \%$ mujeres $(n=7)$ y $22.2 \%$ hombres $(n=2)$, con la siguiente escolaridad: $11.1 \%(\mathrm{n}=1)$ secundaria, $22.2 \%(\mathrm{n}=2)$ carrera técnica, $22.2 \%(\mathrm{n}=2)$ preparatoria, $11.1 \%(\mathrm{n}=1)$ licenciatura y $33.3 \%(n=3)$ posgrado. El estado civil fue $44.4 \%$ solteros $(n=4)$ y $55.6 \%$ casados $(n=5)$.

Uno de los criterios de inclusión fue que los pacientes no cumplieran criterios para psicopatología.

El promedio de edad, peso e IMC se muestra en la siguiente tabla:

Tabla 1

Descripción de las características iniciales de la población de estudio

\begin{tabular}{lccccc}
\hline & N & Mínimo & Máximo & Media & Desv. típ. \\
\hline Edad & 9 & 22 & 52 & 34.11 & 10.914 \\
Peso & 9 & 90.00 & 169.00 & 124.4000 & 26.09526 \\
Imcpre & 9 & 37.00 & 51.00 & 44.1833 & 4.94659 \\
\hline
\end{tabular}

\section{Procedimiento}

Los pacientes candidatos llegaban al servicio de psicología de la clínica de cirugía bariátrica remitidos por el área de cirugía. Se realizaba una evaluación psicológica inicial y se tomaban exámenes de laboratorio que incluían glucosa, triglicéridos, colesterol total, colesterol HDL y colesterol LDL. Si el paciente no cumplía con contraindicaciones absolutas psicológicas ni relativas diferentes a falta de información/conocimiento o expectativas poco realistas de los resultados, se asignaba a intervención cognitivo conductual grupal (véase Anexo 1 - Intervención). El paciente estaba en tratamiento multidisciplinario que incluía nutrición, endocrinología, cardiología, cirugía y rehabilitación física, en conjunto con la intervención psicológica grupal. Al haber concluido la intervención a la que se había asignado volvía a ser sometido a evaluación, y se proponía al equipo multidisciplinario como candidato para cirugía que había completado su preparación para la misma.

\section{Instrumentos}

Los instrumentos que se aplicaron se describen a continuación:

Inventario de Calidad de Vida y Salud (INCAVISA: Sánchez-Sosa, Riveros, y Del Águila, 2009). Evalúa dis- 
tintas áreas que abarcan aspectos diferentes de la vida del paciente: preocupaciones, desempeño, aislamiento, percepción corporal, funciones cognitivas, actitud hacia el tratamiento, tiempo libre, vida cotidiana, familia, redes sociales, dependencia médica y relación con el médico, así como una percepción de bienestar y salud general. Es una escala tipo Likert de 0 (nunca) a 5 (siempre). Tiene una confiabilidad con Alfa de Cronbach de .9337 para vida cotidiana (más alto) y .6831 para actitud ante el tratamiento (más bajo). Muestra adecuadas propiedades psicométricas de consistencia, validez concurrente y test-retest, así como la sensibilidad para distinguir entre diferentes condiciones de salud.

Mini International Neuropsychiatric Interview (MINI: Sheehan et al. 1992) en su versión traducida al español por Ferrando, Bobes, Gibert, Soto, \& Soto (1999). Entrevista corta y estructurada que explora síntomas compatibles con los criterios del DSM IV TR. Versión del Instituto Nacional de Psiquiatría "Ramón de la Fuente Muñiz"; muestra adecuadas propiedades psicométricas para ser utilizada en población mexicana (Heinze, 2000).

\section{Variables}

Puntuación en escala INCAVISA, porcentaje de exceso de peso perdido, peso $(\mathrm{kg})$, IMC, y tiempo que transcurre entre la evaluación inicial y la fecha en que se opera al paciente (meses).

\section{RESULTADOS}

De los pacientes que completaron el estudio, el promedio de IMC inicial fue de $44.18 \mathrm{~kg} / \mathrm{m} 2$ (obesidad grado III o severa). El promedio de peso pre tratamiento fue de 124.4 $\mathrm{kg}$ y el promedio de peso post tratamiento fue de 118.7 $\mathrm{kg}$, logrando en promedio una pérdida de peso de $5.7 \mathrm{~kg}$ en las cinco semanas que duró el tratamiento, mostrando significancia estadística de .001. A su vez, con la pérdida de peso disminuyó el IMC promedio a $42.08 \mathrm{~kg} / \mathrm{m} 2$ con una significancia estadística de .002 . El porcentaje promedio de pérdida de exceso de peso fue de $8.3 \%$, con un rango de $3.7 \%$ a $15.53 \%$.

Tabla 2

Media y Desviación Estándar de peso e IMC pre y post tratamiento

\begin{tabular}{lccc}
\hline & Media & N & Desviación \\
\hline PesoPre & 124.4000 & 9 & 26.09526 \\
PesoPost & 118.7222 & 9 & 26.00984 \\
IMCPre & 44.1833 & 9 & 4.94659 \\
IMCPost & 42.0889 & 9 & 4.77741 \\
\hline
\end{tabular}

$\underline{\text { Tabla } 3}$

Resultados de la Prueba t para peso e IMC pre y post tratamiento

\begin{tabular}{lcccc}
\hline & Media & Desviación & $\mathbf{t}$ & $\mathbf{P}$ \\
\hline Peso & 5.67778 & 3.18700 & 5.345 & .001 \\
IMC & 2.09444 & 1.33895 & 4.693 & .002 \\
\hline
\end{tabular}

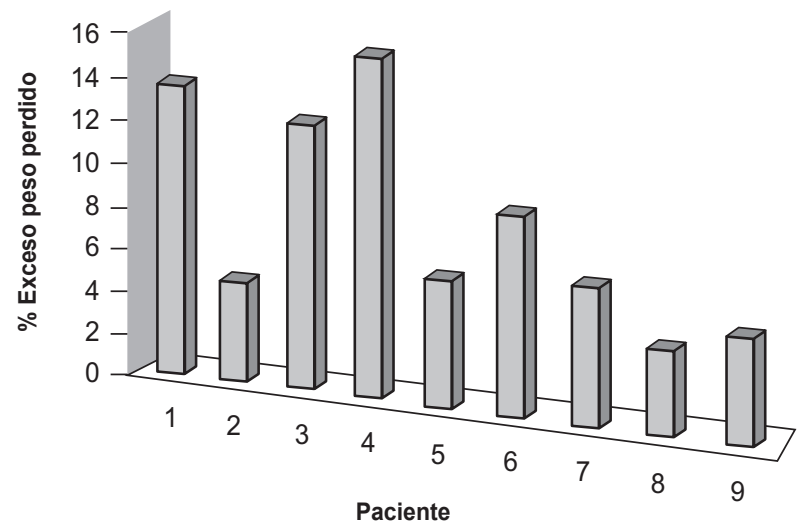

Figura 2. Porcentaje de exceso de peso perdido por paciente

De los nueve pacientes que completaron el estudio, siete se operaron en un promedio de 4.5 meses, con un rango de dos a seis meses, cumpliendo el objetivo de hacer el tiempo pre quirúrgico menor a seis meses.

Se realizó la prueba de Wilcoxon para analizar el cambio en el grado de obesidad. Tres pacientes disminuyeron su grado de obesidad debido a la pérdida de peso; los seis pacientes restantes se mantuvieron en el mismo grado, ninguno subió de grado. A pesar de esto, la disminución de grado de obesidad no fue estadísticamente significativa (p.083).

En cuanto a calidad de vida, se les aplicó en Inventario de Calidad de Vida y Salud (InCaViSa) (Riveros, et al. 2009) identificando las áreas mayormente afectadas, a saber: En primer lugar, percepción corporal (media de 10.87), seguido por relación con el médico (media 7.3), desempeño físico (media de 6) y tiempo libre (media de 5.7). Se analizaron las diferencias pre y post tratamiento con la prueba $t$ de Student para muestras relacionadas, mostrando mejoría significativa en actitud ante el tratamiento (p.04) y en percepción corporal (p.05). 
Tabla 4

Diferencias pre y post de puntuaciones InCaViSa (a mayor puntaje, mayor afectación en el área)

\begin{tabular}{lcccc}
\hline \multicolumn{1}{c}{ Variable } & $\begin{array}{c}\text { Media } \\
\text { Pre }\end{array}$ & $\begin{array}{c}\text { Media } \\
\text { Post }\end{array}$ & $\begin{array}{c}\text { Desviación } \\
\text { Estándar }\end{array}$ & P \\
\hline $\begin{array}{l}\text { Preocupaciones } \\
\text { Desempeño físico }\end{array}$ & 4.85 & 3.71 & 3.7 & .44 \\
$\begin{array}{l}\text { Aislamiento } \\
\text { Percepción } \\
\text { corporal }\end{array}$ & 1.71 & 1.28 & 2.63 & .68 \\
$\begin{array}{l}\text { Funciones } \\
\text { cognitivas }\end{array}$ & 11 & 7.5 & 3.77 & .05 \\
$\begin{array}{l}\text { Actitud al } \\
\text { tratamiento }\end{array}$ & 3.2 & 3.2 & 1.73 & 1 \\
$\begin{array}{l}\text { Familia } \\
\text { Tiempo libre }\end{array}$ & 2.14 & 1 & 1.21 & .04 \\
$\begin{array}{l}\text { Vida cotidiana } \\
\begin{array}{l}\text { Dependencia } \\
\text { médica }\end{array}\end{array}$ & 1.42 & .85 & .78 & .103 \\
$\begin{array}{l}\text { Relación con el } \\
\text { médico }\end{array}$ & 4.57 & 1.71 & 4.67 & .157 \\
\begin{tabular}{l} 
Redes sociales \\
\hline
\end{tabular} & 4.57 & 2 & 3.55 & .685 \\
& 6 & 3.14 & 2.5 & .182 \\
& & 5.5 & 2.5 & .667 \\
& & 1 & 4.3 & .104 \\
\hline
\end{tabular}

Se analizaron las variables peso, IMC y glucosa de manera individual obteniendo los siguientes resultados:

Tabla 5

Resultados pre y post de glucosa

\begin{tabular}{ccc}
\hline Paciente & Glucosa Pre & Glucosa Post \\
\hline 1 & 92 & 81 \\
2 & 90 & 93 \\
3 & 106 & 97 \\
4 & 100 & - \\
5 & 108 & - \\
6 & 167 & 104 \\
7 & 88 & 134 \\
8 & 103 & 90 \\
9 & 82 & - \\
\hline
\end{tabular}

\section{DISCUSIÓN}

La intervención cognitivo conductual grupal de preparación para cirugía bariátrica se asoció a la pérdida de peso y disminución del IMC, así como a la mejoría en percepción corporal y en actitud al tratamiento médico en pacientes con obesidad mórbida sin comorbilidades psiquiátricas. Estos resultados coinciden con los de Leija- Alva y cols. (2011), quienes reportan que pacientes sometidos a intervenciones

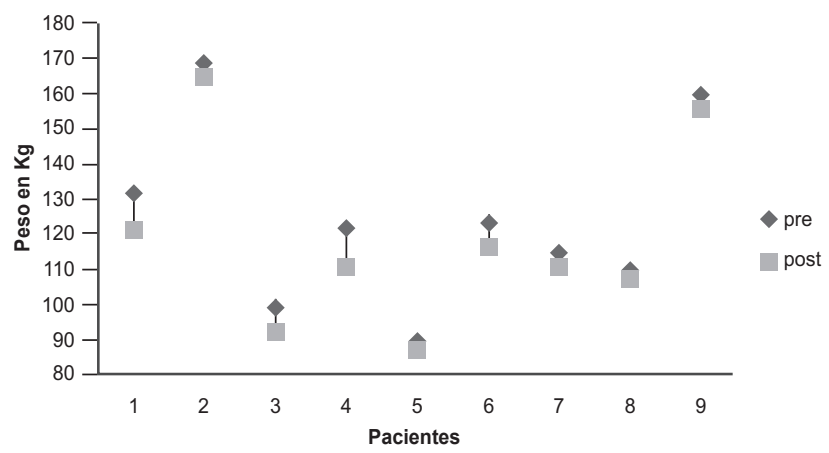

Figura 3. Peso pre y post por paciente

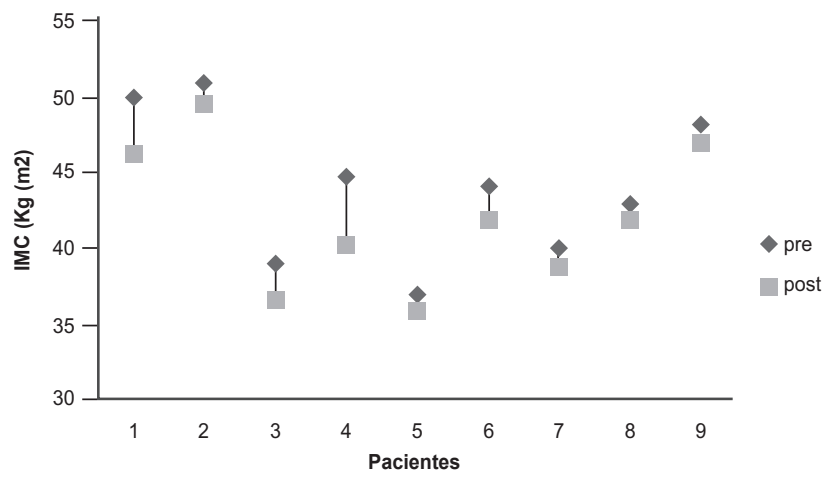

Figura 4. Medidas pre y post de IMC por paciente

cognitivo conductuales para modificación de estilos de vida presentan mejorías en imagen corporal y en hábitos de salud.

Se logró preparar psicológicamente para la cirugía a más pacientes en un mismo periodo de tiempo, optimizando recursos físicos y humanos. La identificación de patrones de alimentación, de barreras que los pacientes han tenido hasta el presente para mantener un peso saludable, la reestructuración de creencias sobre la dieta y el enfoque hacia un estilo de vida saludable fueron aspectos que se manejaron en la intervención. Los resultados obtenidos son similares a los de Yilmaz, et. al. (2011), quienes encontraron que los grupos para manejo de obesidad que utilizan estos elementos han sido útiles para motivar a los pacientes a un cambio en estilo de vida, pérdida de peso, mejoría en calidad de vida e incluso para aumentar la conciencia sobre la enfermedad, descartando opciones de "tratamiento mágico" para su obesidad y privilegiando el cambio en estilo de vida.

Se ha reportado que los pacientes obesos que acuden a terapias grupales muestran mayores niveles de motivación al tratamiento, mejorías en calidad de vida, reducción de depresión y ansiedad, y que esto, a su vez, ayuda en la toma de decisiones sobre someterse a un tratamiento para 
la obesidad (Wild, Herzog, Wesche, Niehoff, Müller, Hain, 2009). Los resultados del presente estudio mostraron una mejoría significativa en la actitud hacia el tratamiento, lo cual aumenta la motivación y adhesión terapéutica, facilitando la pérdida de peso.

Los pacientes perdieron en promedio $8.3 \%$ del exceso de peso en las cinco semanas que duró la intervención. En las guías de la Asociación Americana de Cirugía Metabólica y Bariátrica (AACE, TOS, ASMBS, 2008) se sugiere la pérdida de peso prequirúrgica con el propósito de disminuir el volumen del hígado y mejorar aspectos técnicos de la cirugía, así como para monitorear la capacidad que tiene el paciente de realizar cambios en su estilo de vida. Los pacientes que recibieron esta intervención lograron estos objetivos y posteriormente se practicaron la cirugía.

Se ha descrito que los pacientes que participan en intervenciones grupales pierden mayor peso que quienes lo hacen de manera individual (Orth, Madan, Taddeucci, Coday y Tichansky, 2008). El presente estudio, por ser piloto, no cuenta con grupo de comparación, por lo que se sugiere incluirlo en otras investigaciones.

El objetivo del presente estudio fue promover la pérdida de peso y mejorar la calidad de vida de los pacientes antes de la cirugía; sin embargo, no se estudió la evolución de estos pacientes después de la misma, por lo que también se sugiere incluir un seguimiento a largo plazo que incluya cambios en la calidad de vida, en comorbilidades médicas, en psicopatología, así como en pérdida de peso de los pacientes que reciban intervención grupal de preparación para cirugía bariátrica.

\section{REFERENCIAS}

AACE/TOS/ASMBS, (2008). American Association of Clinical Endocrinologists, The Obesity Society and American Society for Metabolic and Bariatric Surgery Medical Guidelines for Clinical Practice for the Perioperative Nutritional, Metabolic, and Nonsurgical Support of the Bariatric Surgery Patient. Surgery for the Obesity and Related Diseases; 4, 109-184.

Algazi, L. (2000). Transactions in a support group meeting: a case study. Obesity Surgery; 10, 186- 191.

Benotti, P. y Forse, R. (1995). The role of gastric surgery in the multidisciplinary management of severe obesity. American Journal of Surgery; 169, 361-367.

Buchwald, H. (2005). Bariatric Surgery for morbid obesity: health implications for patients, health professionals, and third party payers. Journal of American College of Surgery; 200, 593-604.

Buddeberg-Fischer, B., Klaghofer, R., Sigrist, S. y Buddeberg, C. (2004) Impact of psychosocial stress and symptoms on indication for bariatric surgery and outcome in morbidly obese patients. Obesity Surgery; 14, 361-369.

Colquitt, J., Picot, J., Loveman, E. y Clegg, A.J. (2009) Surgery for morbid obesity. Cochrane Database Systematic Review; (2).

D’ Zurrilla, T. y Goldfried, M. (1971) Problem Solving and Behavior Modification. Journal of Abnormal Psychology; 78, 107- 126.

Hildebrant, S. (1998) Effects of participation in bariatric support group after Roux-en-Y gastric bypass. Obesity Surgery; 8, 535-542.

Kaiser, K., Franks, S., Smith, A. (2011) Positive Relationship between support group attendance and one- year postoperative weight loss in gastric banding patients. Surgery for Obesity and Related Diseases; 7(1): 89-93.

Leija- Alva, G., Aguilera- Sosa, V., Lara- Padilla, E., Rodríguez- Choreño, J., Trejo- Martínez, J., López de la Rosa, M. (2011) Diferencias en la modificación de hábitos, pensamientos y actitudes relacionados con la obesidad entre dos distintos tratamientos en mujeres adultas. Revista Latinoamericana de Medicina Conductual; 1(2), 19- 28.

Marcus, J. y Elkins, G. (2004) Development of a model for a structured support group for patients following bariatric surgery. Obesity Surgery; 14, 103-106.

Miller, W. y Rollnick, S. (2002) Motivational Interviewing: Preparing People for Change. Nueva York: Guilford Press.

Nicolai, A., Ippoliti, C. y Petrelli, M. (2002). Laparoscopic Adjustable gastric banding: essential role of psychological support. Obesity surgery; 12(6), 857-63.

OMS. (2000). Obesity: preventing and managing the global epidemic. Report of a WHO Consultation: Geneva. Recuperado el 19 de noviembre del 2009 de http://www.atividadefisica.pro.br/artigos/WHO\%20obesity.pdf

OMS. (2006). Obesidad y Sobrepeso ¿Qué son la obesidad y el sobrepeso?, Nota Descriptiva No 311 , recuperado el 19 de noviembre del 2009 de http://www.who.int/mediacentre/ factsheets/fs311/es/index.html

Orth, W., Madan, A., Taddeucci, R., Coday, M. y Tichansky, D. (2008). Support Group meeting attendance is associated with better weight loss. Obesity surgery; 18, 391-394.

Riveros- Rosas, A., Sánchez- Sosa, J., Del Águila, M. (2009) Inventario de Calidad de Vida y Salud. México: Manual Moderno.

Sjostrom, L., Lindroos, A.K., Peltonen, M., Tolgerson, J., Bouchard, C., Carlson, B. et. al. (2004). Lifestyle, diabetes, and cardiovascular risk factors 10 years after bariatric surgery. New England Journal of Medicine; 351(26), 2683-93.

Yilmaz, J., Povey, L., Dalgliesh, J. (2011). Adopting a psychological approach to obesity. Nursing Standard; 25(21): 42-46.

Wild, B., Herzog, W.,Wesche, D., Niehoff, D., Müller, B. y Hain, B. (2009). Development of a group therapy to enhance treatment motivation and decision making in severely obese patients with a comorbid mental disorder. Obesity Surgery; published online. 


\section{ANEXO 1}

\section{INTERVENCIÓN}

La intervención cognitivo conductual grupal para candidatos a cirugía bariátrica constó de cinco sesiones grupales realizadas cada siete días; estas sesiones desarrollaban habilidades y brindaban herramientas específicas. Más adelante se describirá cada una de las sesiones. Si el paciente no cumplía con los objetivos especificados, continuaba en preparación pero de manera individual.

A continuación se describen las sesiones de manera más específica:

\section{SESIÓN 1 - EVALUACIÓN}

\section{Objetivos}

En esta sesión se buscaba indagar el nivel de conciencia de los participantes sobre la enfermedad y su percepción de riesgo acerca de ella, así como su motivación, conocimiento y expectativas con respecto a la cirugía. Se buscaba identificar factores cognitivos y conductuales que pudieran interferir con el proceso de la cirugía bariátrica (por ejemplo, hábitos específicos, creencias irracionales), trastornos de la conducta alimentaria (atracón, ingesta emocional, síndrome de alimentación nocturna), redes de apoyo social, habilidades para manejo del estrés, y niveles de autocontrol y autoeficacia percibida. También se buscaba identificar si existía algún factor psicológico contraindicado para la cirugía bariátrica (toxicomanías, adicción al alcohol, trastornos psiquiátricos no controlados).

Los datos obtenidos en esta primera sesión arrojaron un perfil inicial del paciente y se utilizaron como medición pre tratamiento.

\section{Procedimiento}

Se realizó una entrevista semi estructurada para candidatos a cirugía bariátrica y una entrevista MINI Neuropsychiatric International Interview con el objetivo de identificar la psicopatología. Se tomaron medidas antropométricas y se aplicó el Inventario de Calidad de Vida y Salud (InCaViSa) (Riveros- Rosas, Sánchez-Sosa, Del Águila, 2009).

Si el paciente cumplía con los criterios de inclusión se pedía su consentimiento y se ingresaba al estudio.

\section{SESIÓN 2 - CIRUGÍA BARIÁTRICA}

\section{Objetivos}

Incrementar niveles de conocimiento sobre obesidad, riesgos, causas y tratamientos; aumentar la percepción de riesgo y la conciencia de la enfermedad.
Generar conciencia de enfermedad (que el paciente aceptara la condición de obesidad como una enfermedad, y por lo tanto asumiera la responsabilidad sobre el tratamiento).

Instruir al paciente sobre los tipos de cirugía bariátrica que existen, los procedimientos y cambios de estilo de vida que debería realizar si se sometía a este tratamiento, y sobre planeación de la compra y administración de suplementos alimenticios.

Aumentar la percepción de control y motivación, así como disminuir la ansiedad provocada por la incertidumbre.

Promover el aprendizaje vicario por medio de compartir experiencias entre pacientes.

\section{Procedimientos}

Se realizaba una sesión de psicoeducación grupal con los pacientes candidatos a cirugía bariátrica; se brindaba información sobre la obesidad (causas, riesgos), tratamientos y sobre la cirugía bariátrica. Se llevó a cabo un balance sobre la decisión de practicarse o no, la cirugía bariátrica, así como la identificación de conductas que debían modificarse y las posibles alternativas existentes para ello.

\section{SESIÓN 3 - ESTILOS DE VIDA SALUDABLES}

\section{Objetivos}

Instruir al paciente para que conociera los cambios en estilo de vida que debería adoptar previo a la cirugía, poniendo de presente que ésta no es una solución "mágica" para el problema de la obesidad. Se hacía énfasis en que si no modificaba sus hábitos, no lograría bajar el peso deseado ni podría mantener el que había alcanzado. Adicionalmente, se buscaba generar en el paciente la capacidad de identificar hábitos saludables y no saludables que él mismo presentaba, así como los que principalmente podrían interferir en el proceso pre y post quirúrgico. Por último, se llevaba al paciente a identificar aquellos hábitos que debían cambiarse, las razones para ello y la forma como debería hacerlo (planeación), así como las barreras que percibía para lograrlo y cómo podría superarlas.

\section{Procedimientos}

Psicoeducación sobre estilos de vida saludables, utilizando elementos de entrevista motivacional (Miller \& Rollnik, 2002), Es un tipo de entrevista clínica centrada en el paciente, que ayuda a explorar y resolver ambivalencias acerca de una conducta o hábito insano con el objeto de promover cambios hacia estilos de vida más saludables. A través de esta estrategia se ayudaba a que el paciente 
tomara la decisión de cambiar. Se tenían en cuenta los intentos anteriores por lograr estos cambios y las razones por las que no funcionaron.

Se incluyeron técnicas conductuales para facilitar el cambio en estilo de vida:

Control de Estímulos/Modificación ambiental: evitar comprar alimentos no permitidos en el plan de alimentación, tener alimentos saludables preparados en casa, comer en un solo lugar sin realizar otras actividades al mismo tiempo.

Preparar redes de apoyo: el paciente identificaba quiénes eran sus redes de apoyo y de qué manera podrían ayudarle para lograr el cambio en su estilo de vida.

Conductas Incompatibles: identificar y proponer actividades que el paciente podría llevar a cabo cuando sintiera "ansiedad por comer".

Estructurar patrones de alimentación: planeación de horarios, lugares y tipos de alimentos.

Identificar/controlar ingesta emocional y recreativa: identificar si la presentaba; en caso afirmativo, realizar un plan de acción con las actividades que se enlistaron anteriormente y probar cómo funcionaban las diferentes alternativas.

Comenzar rutina de ejercicio: que el paciente realizara su actividad física preferida y llevara un registro con los días en que la practicaba y el tiempo que le dedicaba.

Identificación de pensamientos disfuncionales para el cambio de estilo de vida y aprendizaje sobre cómo realizar un autodebate (más en detalle en sesiones posteriores).

Automonitoreo: alimentos, horarios de alimentación, emociones al comer, ejercicio.

\section{SESIÓN 4 - ENTRENAMIENTO EN SOLUCIÓN DE PROBLEMAS ENFOCADO EN CAMBIOS EN ESTILO DE VIDA}

\section{Objetivos}

Brindar al paciente herramientas para análisis y solución de problemas relacionados con ingesta emocional y recreativa.

Proponer soluciones para enfrentar estas situaciones, así como los problemas que hubiera tenido hasta el momento con el cumplimiento de la dieta y la actividad física.

\section{Procedimientos}

El entrenamiento en solución de problemas propone un esquema orientado a que el paciente aprenda a resolver diversos problemas actuales y futuros, con apoyo del Manual de Técnica de Solución de Problemas basado en la propuesta de D'Zurilla \& Golfried (1971). Recalcar la importancia de enfrentar los problemas y la asociación que puede existir entre la baja capacidad de resolverlos y comer; aumentar sensación de control sobre ingesta emocional y recreativa.

Meta de la sesión: Que el paciente lograra identificar y operacionalizar los problemas que se le hubieran presentado en el cambio en estilo de vida, que pudiera proponer soluciones, escoger alguna con base en un análisis del costo/ beneficio de las mismas, y que realizara el plan de acción para poner en práctica esa solución.

\section{SESIÓN 5: AUTOREGULACIÓN}

\section{Objetivo}

Instruir al paciente para que aprendiera a relajar su cuerpo, de tal modo que lograra identificar la diferencia entre situaciones de estrés/tensión y de relajación. Adicionalmente, se buscaba aumentar en el paciente la percepción de control sobre las respuestas del cuerpo, específicamente sobre la respuesta de relajación y frecuencia cardiaca. Brindar una herramienta para cuando el paciente sintiera ansiedad por comer, ansiedad ante estudios exploratorios $\mathrm{y}$ ansiedad pre quirúrgica.

Que incluyera dentro de su repertorio habitual de conducta esta técnica de relajación para que pudiera lidiar con los diferentes estresores que se le presentaran.

\section{Procedimientos}

Se brindó una psicoeducación sobre componentes cognitivos y fisiológicos del estrés. Se realizó un entrenamiento en respiración diafragmática grupal y en imaginación guiada.

Se le explicaba que esto podía realizarlo cuando sintiera tensión, ansiedad, estrés y esas sensaciones le generaran ganas de comer. Se le indicaba que era necesario practicar en casa para perfeccionar la respuesta y producir el efecto deseado para la cirugía.

\section{BLOQUE 5: REESTRUCTURACIÓN COGNITIVA}

\section{Objetivo}

Identificar creencias irracionales/distorsiones cognitivas del paciente que interfieren con la conducta de seguir el plan de alimentación y de realizar actividad física. Entrenar al paciente en el autodebate y la reestructuración cognoscitiva, enfocados al cambio de algunas creencias por otras más adaptativas, que le ayudarían a seguir el plan de alimentación y de actividad física.

\section{Procedimientos}

Al identificar las creencias, el psicólogo debía llenar el registro de pensamiento junto con el paciente. De igual manera, debía enseñar, a través del determinismo recíproco, cómo los pensamientos influyen tanto en las emociones 
como en las conductas y hacer hincapié en que si se logra cambiar el pensamiento, es posible cambiar la emoción y la conducta. Las creencias que se buscaba cambiar eran del tipo "ya rompí la dieta, voy a romperla bien", "hoy estoy cansado(a), no haré ejercicio", "ya voy a operarme, hasta entonces seguiré la dieta". En ocasiones, estos pensamientos surgían en situaciones específicas, pero de igual manera influían en la conducta; un ejemplo de esto era: "Debo terminarme todo lo que hay en el plato" (pensamiento); la conducta será que termine todo lo que hay en el plato; si el paciente aprendía a debatir estas creencias, podría modificarlas y por lo tanto, también cambiaría su conducta. Se le pedía que identificara algunas creencias desadaptativas y que hiciera el ejercicio de autodebate. Posteriormente se le pedía que practicar en casa. En el manual se incluía un registro de pensamientos; debía realizar un registro constate de éstos hasta la siguiente sesión.

\section{SEGUNDA EVALUACIÓN}

Se realizó otra entrevista con el paciente de manera individual para obtener retroalimentación sobre qué había aprendido el paciente, para aclarar las dudas y volver a realizar la batería de pruebas psicológicas. En caso de que el paciente no hubiera cumplido los objetivos, continuaría en tratamiento psicológico individual. En caso de que los hubiera cumplido, se presentaría al equipo multidisciplinario como candidato a cirugía por parte de psicología. 